\title{
La Tecnología en las Organizaciones Complejas
}

\author{
Carlos Osorio M.*
}

* Máster en Historia y Filosofía de las Técnicas - Profesor Asistente Unidad de Gestión Tecnológica - Escuela de Ingeniería Industrial y Estadística - Universidad del Valle Santiago de Cali, Colombia.

E-mail:carosori@yahoo.com

Fecha de recepción: Octubre 5 de 2002

Fecha de aprobación: Octubre 17 de 2003

\section{RESUMEN}

El presente trabajo intenta caracterizar, muy brevemente, la forma como la tecnología hace parte de las organizaciones, partiendo de los modelos organizacionales desarrollados a lo largo del siglo XX. De organizaciones cerradas, a otras abiertas tipo red, la tecnología ha pasado de ser el centro del determinismo tecnológico, de dictar toda la base de los procesos internos en una organización, a un espacio de negociación entre las personas que hacen parte de un determinado sistema tecnológico. En este sentido, las organizaciones más horizontales, que han empezado a emerger desde los años 80 del siglo $x x$, reconocen la tecnología como algo menos 
objetivo, algo permeado por los valores y la cultura de quienes coordinan y participan en ellas.

\section{Palabras clave:}

Organizaciones. Sistema tecnológico. Determinismo tecnológico

\section{ABSTRACT}

The present work tries to characterize, very shortly, the form like the technology makes part of the organizations, leaving of the organizational models developed along the $X X$ century. In the closed organizations, the technology has passed of being the center of the technological determinismo, to a negotiation space among people that make part of a certain technological system. In this sense, the organizations, more horizontal, recognize the technology like something less objective, something permeado for the values and the culture of those who coordinate and they participate in them.

\section{Keywords:}

Organizations. Technological system. Technological determinism.

\section{INTRODUCCIÓN}

En el presente trabajo se abordará el tema de la organización, presentando brevemente la forma como están definidas las organizaciones, para pasar a caracterizar la relación con la tecnología, a partir de la estructura de la organización. Para ello seguiremos los planteamientos de aquellos que podemos denominar teóricos de la organización (administradores, psicólogos, sociólogos, economistas, entre otros).

La relación de la tecnología con la estructura organizacional ha sido bien estudiada por teóricos de las organizaciones (Thompson, 1.993; Gerwin y Kolodny, 1.992; Sankar, 1.991; Woodward, 1.976; Scott 1.992; Hall, 1.977). Gran parte de estos trabajos analiza las diversas formas con las que se ha pensado la tecnología, de acuerdo a los tipos de organización. Resulta relevante que se asuma la estructura de una organización, como directamente dependiente de la tecnología. Y aunque no se agota en esta definición, se señala con frecuencia que si la tecnología determina la estructura, entonces la selección de una estructura organizacional apropiada debe hacerse con base en las características de la tecnología o tecnologías particulares.

Finalmente, con la revisión de algunas posiciones representativas de los teóricos de la organización, sobre la relación tecnología y organización, esperamos que se consolide una introducción general del asunto. Ello no excluye algunos elementos reflexivos acerca del papel de las personas en esta relación; se trata de una cierta preocupación hermenéutica, en el sentido en que Mitcham (1.989: 82-83) lo ha señalado para la tecnología: la búsqueda por penetrar en su significado, definiendo sus vínculos. La hermenéutica se emplea en este contexto siguiendo el lugar que ocupa en todas las ramas humanísticas, como una actividad sin reglas, intuitiva y tácita, pero no-arbitraria. Es un intento por lograr un conocimiento comprensivo, más que una explicación lógica y como tal, no se dirige hacia asuntos que son indiferentes o externos al yo. La interrogación hermenéutica permite entrar en la tecnología y la organización, en su mutua relación, en la búsqueda de aprehenderlas, interpretarlas, entenderlas, partiendo de que se trata de conocimientos históricamente condicionados.

\section{MODELOS ORGANIZACIONALES}

Existen organizaciones desde que existen grupos humanos que coordinan actividades, no obstante el carácter formal, relacionado con la tecnología, se impone específicamente en las sociedades capitalistas. Mo toda colectividad es considerada como una organización formal. El criterio de definición, es la existencia de procedimientos para movilizar y coordinar los esfuerzos de diversos subgrupos, generalmente especializados, con miras a alcanzar objetivos comunes (Blau, 1.974: 469). 
Con base en los principios de la cibernética de sistemas complejos, algunos teóricos de las organizaciones han identificado tres grandes modelos para referirse al comportamiento de éstas: el modelo racional, el modelo natural y el modelo de sistema abierto (Scott, Op. cit.: 28). Veamos cada uno de estos modelos, antes de analizar el papel de la tecnología en los diversos tipos de organización que los originaron.

\subsection{El modelo racional}

El término racional, en este contexto, es usado en estrecho sentido de "técnico" o "racionalidad funcional" y se refiere a una serie de acciones organizadas de tal forma que siguen una metas predeterminadas que conllevan a una máxima eficiencia (Scott, 1.992: 29-30).

Una organización tiene un sistema cerrado, basado en una estructura racional, cuando puede fijar las circunstancias presentes, esto es, cuando las variables y relaciones implicadas son suficientemente pocas o reducidas para que las comprendamos y para que tengamos control sobre todas ellas o podamos predecirlas de manera confiable (Thompson, Op. cit.: 6). El comportamiento de las organizaciones es visto como ejecución de acciones para utilizar y coordinar agentes. El lenguaje empleado en este tipo de organizaciones connota la imagen de cálculo racional, con términos como: información, eficiencia, optimización, implementación y diseño. Por otro lado, las interacciones dentro del sistema de administración tienden a ser verticales entre superior y subordinado, hay una firme jerarquía de mando, para el cual las decisiones individuales hechas en el contexto organizacional requiere de términos como: constricción, autoridad, reglas, directivas, jurisdicción, ejecución de programas, coordinación; todas ellas indican que la racionalidad del comportamiento en organizaciones de este tipo, tiene lugar en límites claramente especificados (Scott, Op. cit.: 30).

La racionalidad de esta clase de organización, reside en su propia estructura, más que en los participaciones individuales; en reglas que aseguran el comportamiento de los participantes en vías calculadas para llevar a cabo objetivos deseados; en disposiciones de control, que evalúan la ejecución y detectan desviaciones; en la revisión de sistemas que motivan a los participantes para realizar tareas preescritas; y en un set de criterios con los cuales los participantes son seleccionados, reemplazados y promovidos (ibid.: 49). Esta organización sin gente, ideal para los sistemas de expertos que colocan las decisiones en función de máquinas y procesos informatizados, ha sido considerada como una nueva utopía (Boguslaw, 1.965).

Buena parte de la literatura sobre organizaciones, emplean supuestos del sistema cerrado, dentro del modelo racional. Los ejemplos quizás más conocidos son los de la administración científica de Taylor (1.911), y el modelo burocrático de Weber (1.946). En el caso de Weber, la capacidad de cambio es posible de afrontar, con un orden social legítimo, a partir de un esquema común de valores que se traduce en control social y en observancia de las normas dentro de una sociedad. En otras palabras, la estructura de autoridad o de dominación es el núcleo de la organización social y las estructuras de autoridad pueden diferenciarse atendiendo a los sistemas de valores que las legitiman. El análisis emprendido por Weber le llevó a considerar que en el mundo moderno, las grandes colectividades que se enfrentan con tareas administrativas complejas, son las que con mayor probabilidad llegan a organizarse burocráticamente.

\subsection{El modelo natural}

En este caso, se considera que las organizaciones mantienen una interacción permanente con el medio ambiente, lo que no permite considerarlas como entidades autónomas, por consiguiente están sujetas a otras unidades $u$ organizaciones complejas, de las cuales dependen (Barnard, 1.938). De este modo, las metas específicas que 
realizan las organizaciones, frecuentemente están indeterminadas o distorsionadas por energías dependientes de la consecución de los objetivos del sistema. Mientras las organizaciones del modelo racional hacen hincapié en aspectos estructurales normativos, las organizaciones de sistema natural tienen más énfasis en aspectos de comportamiento de los participantes (Scott, Op. cit. : 73).

Muchos de los teóricos del modelo natural siguieron la perspectiva del análisis funcional, sobre la premisa de que existían necesidades que debían ser satisfechas para sobrevivir, de ahí que se prestaba atención en descubrir los mecanismos por los cuales esas necesidades eran satisfechas (Bernard, Selznick, Parsons). Por otro lado, consideraban que el medio ambiente estaba asociado con el sistema natural, en donde, con frecuencia, era percibido como algo enemigo o como una fuente de presiones y problemas; la organización era vista tanto como una capitulación por un medio ambiente tiránico y hostil, como el precio pagado para sobrevivir (Selznick, 1.957); mientras que en el caso de Parsons (1.960), la organización es un subsistema, en donde el medio ambiente es fuente de resistencias y elemento estabilizador que la sostiene y legitima en su misión.

Las organizaciones de sistema natural tienen sistemas administrativos mejor adaptados a las condiciones cambiantes del medio ambiente y de la tecnología. En el sistema natural u orgánico predomina el ajuste y la redefinición continua de las tareas individuales a través de la interacción con otros, en él tienen mucho peso las actitudes y actividades individuales; es una organización que funciona más como una red, en lugar de un control jerárquico, con interés en comunicaciones laterales más que verticales, con una amplia dispersión del poder que depende de la experiencia técnica y del conocimiento, y no tanto en posiciones jerárquicas; los procesos de toma de decisiones son más innovadores y basados en el juicio y se orientan hacia la solución de problemas, más que a la consecución de metas de actuación (Kast y Rosenzweig, 1988: 235).

\subsection{El modelo abierto}

Las organizaciones de sistema abierto se relacionan teóricamente con la cibernética y la teoría de la información, desarrollada en la ingeniería a través de la investigación de operaciones y de manera amplia con la teoría general de sistemas (Bertalanffy, 1.976). Según Wiener (1.969), una organización tiene que ser considerada como algo en la cual hay una interdependencia entre las diferentes partes organizadas, pero esta interdependencia presenta grados. Las organizaciones de sistema abierto son concebidas como sistemas cibernéticos, es decir, capaces de su propia regulación, en términos de alguna externalidad preescrita como objetivo o criterio. Un sistema abierto es aquel capaz de mantenerse sobre la base de canalizar sus recursos del medio ambiente; toma energía del medio ambiente y adquiere entropía negativa.

Sin embargo, una organización al ser parte de un sistema social, puede ser vista como una coalición de grupos e intereses, obteniendo alguna cosa de la colectividad, pero interactuando con otros, cada uno con sus propias preferencias y objetivos (Scott, Op. cit.: 82). Las organizaciones humanas pueden ser concebidas como sistemas multicefálicos, capaces de recibir información, tomar decisiones y dirigir acciones; por lo que la interacción no es un asunto mecánico.

\section{ESTRUCTURA ORGANIZACIONAL Y TECNOLOGÍA}

Tecnología, según los teóricos de las organizaciones incluye no sólo el hardware usado en la ejecución del trabajo, también las herramientas y los conocimientos de los trabajadores, al igual que las características de los objetos con los cuales el trabajo es ejecutado. Mo sobra señalar que hay más de una definición para 
la tecnología, por ejemplo, la tecnología como artefacto, como ciencia aplicada y como sistema tecnológico (Osorio, 2002). También hay que aclarar que se presenta una permanente superposición de términos empleados en el análisis organizacional, tales como: tecnología, sistema técnico, tarea medioambiental, medioambiente. Medioambiente es el término más integrador, incorpora aspectos políticos, tecnológicos e institucionales del contexto organizacional. La tecnología es vista como el proceso que combina el conocimiento intelectual con el físico, por medio del cual los materiales son transformados en outputs (Scott Op. cit.: 228). Para una organización, el medio ambiente no es sólo una fuente de inputs y de recibimiento de outputs, también la tecnología es percibida como parte del medio ambiente, debido a que la mayoría de las organizaciones no inventan sus tecnologías; de ahí la frecuente superposición de expresiones (ibid).

Si asumimos la tecnología como la base de la estructura organizacional, el papel de la gerencia, descle este punto de vista, es pasivo; la estructura debe seguir los arreglos que la tecnología decide (Gerwin, 1.981). La escogencia de una estructura organizacional apropiada, que sigue un claro establecimiento acerca de las características de la tecnología particular o tecnologías a implementar, se le conoce como determinismo tecnológico. De manera más amplia, se entiende por determinismo tecnológico, la creencia de que las fuerzas técnicas determinan los cambios sociales $y$ culturales (Hughes, 1996: 118). EI determinismo tecnológico sería la base del diseño de las organizaciones de manufactura, particularmente en las tecnologías de procesos.

En una tecnología de proceso continuo de una planta petroquímica, por ejemplo, el proceso para refinación del crudo en productos, sigue una formulación química específica que dicta la secuencia de operaciones que tendría lugar en la organización. La translación de esa secuencia de pasos dentro de una disposición de equipamento técnico -pipas, válvulas, bombas- sigue una determinada solución que es asumida como la vía óptima de llevar a cabo los procesos de producción (Gerwin y Kolodny, 1.992: 142).

De un mapa de soluciones técnicamente determinadas, sale el diseño técnico esencial del sistema de producción. La organización del trabajo, el diseño de puestos, los arreglos organizacionales y otros sistemas de soporte (por e.j. el sistema social total); dependen de la solución técnica que hace posible el sistema total de producción. En el determinismo tecnológico, el sistema social se adapta al sistema técnico y las personas no son más que extensiones del sistema, su función es completar el diseño técnico (ibid.: 143).

Las diferentes formas de estructura que han existido en las organizaciones y que han sido dominantes en el entorno manufacturero, particularmente desde la perspectiva Morteamericana, se resumen en las Tablas Mo. 1 y 2 , según Gerwin y Kolodny (Op. cit. : 133, 137).

Mo vamos a desarrollar las características de cada una de estas organizaciones señaladas en las tablas, simplemente resaltaremos algunas en función de su relación con la tecnología. En la forma funcional, por ejemplo, el orden organizacional fue diseñado alrededor de la división del trabajo, en donde las tecnologías de trabajo en equipo, en una fábrica tradicional que use, por ejemplo, tornos, fresadoras, taladros, molinos y brilladoras, etc., eran organizadas como una función común. Esta clase de organizaciones se caracteriza por una concepción del desarrollo tecnológico basada en la permanente adquisición de maquinaria, más que por la consulta de un sistema de expertos; lo que las lleva a mantenerse tecnológicamente actualizadas, asumiendo que la tecnología es básicamente maquinara.

Sin embargo, los modelos funcionales son una estructura pobre para organizaciones que pretenden respuestas rápidas frente a incertidumbres del entorno, ya que su preocupación es establecer controles de producción programados (ibid.: 133-134). 


\begin{tabular}{|c|c|c|c|}
\hline Período & $\begin{array}{c}\text { Modelo y } \\
\text { Características }\end{array}$ & $\begin{array}{c}\text { Énfasis } \\
\text { Fundamental }\end{array}$ & $\begin{array}{l}\text { Características } \\
\text { Intrínsecas }\end{array}$ \\
\hline \multirow[t]{2}{*}{ Hasta 1950} & Funcional & Manufactura & \\
\hline & $\begin{array}{r}\text { Eficiente } \\
\text { Desarrollo }\end{array}$ & & $\begin{array}{l}\text { División del trabajo } \\
\text { Economías de escala } \\
\text { Caminos de Carreras verticales }\end{array}$ \\
\hline \multirow[t]{2}{*}{ 1950s - 1970s } & Divisional & Mercadeo & \\
\hline & $\begin{array}{r}\text { Descentralizada } \\
\text { Orientación externa } \\
\text { Diversificada }\end{array}$ & & $\begin{array}{l}\text { Autonomía local } \\
\text { sensibilidad local } \\
\text { Dirección general }\end{array}$ \\
\hline $1970 s-1980 s$ & $\begin{array}{l}\text { Mixta } \\
\text { (Proyecto/Matriz) } \\
\text { Adaptable } \\
\text { Compleja } \\
\text { Creciente variedad } \\
\text { Dos o más } \\
\text { orientaciones }\end{array}$ & $\begin{array}{l}\text { Nuevos productos } \\
\text { Procesamiento de } \\
\text { la información }\end{array}$ & $\begin{array}{l}\text { Comunicaciones laterales } \\
\text { Alta interacción } \\
\text { Caminos de carreras múltiples } \\
\text { Equipos }\end{array}$ \\
\hline
\end{tabular}

Tabla 1: Formas Organizacionales. Parte I, hasta los años 1980

\begin{tabular}{|c|c|c|c|}
\hline Período & $\begin{array}{c}\text { Modelo y } \\
\text { Características }\end{array}$ & $\begin{array}{c}\text { Énfasis } \\
\text { Fundamental }\end{array}$ & $\begin{array}{c}\text { Características } \\
\text { Intrínsecas }\end{array}$ \\
\hline 1970s & $\begin{array}{l}\text { Enfoque en el producto } \\
\text { Flexibilidad } \\
\text { Tamaño Pequeño } \\
\text { Autonomía relativa } \\
\text { Orientación hacia el flujo }\end{array}$ & $\begin{array}{l}\text { Tecnología de } \\
\text { Producción } \\
\text { Tiempo corto } \\
\text { de ciclo }\end{array}$ & $\begin{array}{l}\text { Computarización } \\
\text { Automatización } \\
\text { Trabajo en grupo } \\
\text { Entrenamiento } \\
\text { Multifuncional }\end{array}$ \\
\hline 1970s & $\begin{array}{l}\text { Sociotécnico } \\
\text { Propia regulación } \\
\text { Incremento de la variedad } \\
\text { Manejo de valores } \\
\text { Orientación en sistemas } \\
\text { Alanza sociotécnia }\end{array}$ & $\begin{array}{r}\text { Adaptabilidad } \\
\text { Compromiso de } \\
\text { la gente }\end{array}$ & $\begin{array}{l}\text { Gerencia limitada } \\
\text { Crecimiento y Aprendizaje } \\
\text { Grupos autónomos } \\
\text { Diseño participativo }\end{array}$ \\
\hline $1980 s$ & 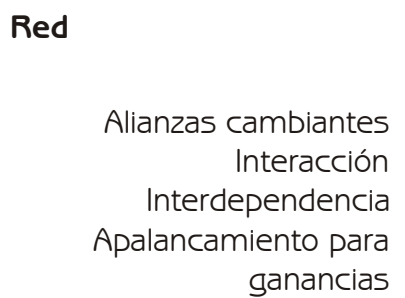 & $\begin{array}{r}\text { Tecnología } \\
\text { de información } \\
\text { Trabajo de } \\
\text { conocimiento }\end{array}$ & $\begin{array}{l}\text { Relaciones laterales } \\
\text { Comunicaciones } \\
\text { Multifunciones limitadas } \\
\text { Redes de información }\end{array}$ \\
\hline $1985+$ & $\begin{array}{r}\text { Integrativo } \\
\text { Número mediano } \\
\text { Diseño socioténico } \\
\text { Filosofía compartida } \\
\text { Plantas dentro de plantas }\end{array}$ & $\begin{array}{r}\text { Gerencia de negocios } \\
\text { Gente, calidad, } \\
\text { tecnología y } \\
\text { racionalización de la } \\
\text { información }\end{array}$ & $\begin{array}{l}\text { Diseño continuo } \\
\text { Diseño participativo } \\
\text { Límites colapsados } \\
\text { Procesamiento paralelo }\end{array}$ \\
\hline
\end{tabular}

Tabla 2: Formas Organizacionales. Parte II, desde los años 1970 
Tanto los modelos divisionales, que surgieron a partir de los funcionales dentro de las grandes corporaciones, así como los que dependían de una estructura matricial con la capacidad de manejar simultáneamente la gestión funcional y las orientaciones de proyectos; dieron origen a organizaciones que respondían al modelo tipo racional, expresado antes; por consiguiente, son organizaciones relativamente cerradas, con sistemas administrativos jerárquicos, combinados con cierto nivel de descentralización (más en organizaciones departamentalizadas y matriciales); y una tendencia a la alta profesionalización, la cual responde a una gran complejidad técnica asociada con una gran complejidad estructural (Scott, Op. cit. : 226256).

Las organizaciones más relacionadas con el medio ambiente, tanto la natural como la de sistema abierto, parten de una cierta crítica al modelo racional, aquella que considera que la tecnología es una construcción social, la que cuestiona que estemos abandonados a una lógica inexorable del determinismo tecnológico; aboga por una combinación de lo que es técnicamente posible y políticamente aceptable (Scott: 243). Es decir, si para los teóricos de la década de los 60, como Perrow, Woodward y Thompson, la tecnología era tratada como algo objetivo; teóricos más recientes han hecho hincapié en que los diferentes actores situados en las organizaciones, tienen puntos de vista divergentes sobre una determinada tecnología. Es más, cuando se habla de sistema técnico, se considera que es muy grande la oportunidad para que las fuerzas sociales y políticas operen, (Scott, Op. cit. : 246).

Tales características más amplias acerca de la relación tecnología y organización, se encuentran en las organizaciones de modelo de red y de diseño sociotécnico, que aparecen en la tabla 2 . En las organizaciones en red, surgidas en la década de los 80 , se considera que constituyen un modelo organizacional muy flexible (Gerwin y Kolodny, Op. cit.: 137-138); utilizan tecnologías de comunicación y de información, así como trabajadores especializados o de conocimiento, especialmente para aquellas tareas de investigación y desarrollo. Algunas de estas organizaciones son de tamaño pequeño y basan su estrategia en alianzas con otras empresas, consiguiendo así una mayor eficacia en la cadena de valor.

Si para la concepción determinista, que ha imperado en gran parte en las formas organizacionales, la tecnología es la base del diseño organizacional, desde una concepción sociotécnica se aboga por una perspectiva alternativa. Se sugiere que las organizaciones deberían ser diseñadas por personas que están en roles complementarios a las máquinas, antes que ser extensiones de las mismas (Jordan, 1.979). Se parte del principio que se está siempre en más de una vía para diseñar un sistema técnico y algunas de las alternativas no solo conducen a unas mejores soluciones sociales del sistema sino también a un resultado aún más efectivo del diseño total de la organización.

\section{CONCLUSIONES}

La forma como es vista la relación tecnologíaorganización depende mucho del tipo de estructura organizativa. Si se trata de organizaciones racionales, de modelo cerrado, que imperaron en los modelos funcionales, incluso divisionales; la tecnología es la que decide sobre la estructura, bajo un sistema de asignación de los agentes, el cual genera la eficiencia de la organización; más que en las decisiones de los mismos. La actuación de los agentes depende de la estructura y de sus metas de actuación; es un sistema burocrático, que controla las contingencias del ambiente; es pues, una tecnología social, lo más perfecta y rutinaria posible en la consecución de sus objetivos preestablecidos.

Una organización de tipo natural, además de reconocer algunos aspectos de la organización racional, se caracteriza por su capacidad de asociarse con el medio ambiente. Se trata de una tecnología social más comprensiva, que da cierto 
margen de actuación a los agentes, pero bajo líneas de comportamiento relativamente trazados. Por último, hemos visto la organización abierta, basada en modelos cibernéticos, pero tratándose de una tecnología social, contiene elementos débilmente conectados, lo que genera una cierta autonomía en las personas que lo integran. Hablamos aquí de personas más que de agentes.

Desde los modelos de organizaciones de tipo natural y especialmente de tipo abierto, con incidencia desde los años 70, surgieron las de tipo tipo red o tipo sociotécnico. Ésta última es la que permite, al interior de las organizaciones, una mayor participación de las personas en las elecciones tecnológicas.

\section{BIBLIOGRAFÍA}

BARMARD, C. THE FUMCTIONS OF THE EXECUTIVE. Harvard University Press, Cambridge, 1938.

BERTALAMFFY, L. V. TEORÍA GEMERAL DE LOS SISTEMAS. Fondo de Cultura Económica, México, 1976.

BLAU, P. M. "Organizaciones: Teorías", en: D. SILLS, (ed.), Enciclopedia internacional de las ciencias sociales. Aguilar, Madrid, V. 7, 1974, pp. 469-474.

BOGUSLAW, R. THE MEW UTOPIAMS: A STUDY OF SYSTEM DESIGM AND SOCIAL. Prentice Hall, Englewood Cliffs, M. J., 1965.

GERWIM, D. Y KOLODMY, H. MAMAGMENT OF ADVAMCED MAMUFACTURIMG TECHMOLOGY. STRATEGY, ORGAMIZATIOM \& IMMOVATION. John Wiley \& Sons, Inc., Mew York, 1992.

GERWIM, D. "Relationships Between Structure and Technology", en: P. MYSTROM, y W. STARBUCK. Handbook of organizational designs. Oxford University Press, Mew York, vol. 2, 3-38, 1981.

HALL, R. ORGAMIZATIOMS. STRUCTURE AMD PROCESS. Prentice-Hall, Inc., M. J. , 1977.

HUGHES, T. P. "Technological Momentum", en: M. ROE SMITH, Y L. MARX (eds.), Historia y determinismo tecnológico. Alianza Editorial, Madrid, 1996.
JORDAM, M. "Allocation of Functions Between Man and Machines in Automated Systems", en: L. DAVIS y J. TAYLOR. Design of jobs. Goodyear Publishing, Santa Monica, 1979, 6-11,

KAST, F. Y ROSEMZWEIG, J. ADMIMISTRACIÓM EM LAS ORGAMIZACIOMES. EMFOQUE DE SISTEMAS Y DE COMTIMGEMCIAS. McGrawHill/Interamericana de México S. A., México, 1988.

MITCHAM, C. ¿QUÉ ES LA FILOSOFÍA DE LA TECMOLOGÍA?, Anthropos, Barcelona, 1989.

OSORIO, C. "Enfoques sobre la Tecnología". Revista de Ciencia, Tecnología y Sociedad + Innovación, (\# 2). Enero-abril 2002,

PARSOMS, T. STRUCTURE AMD PROCESS IM MODERM SOCIETIES. Free Press, Glencoe, 1960.

PERROW, CH. SOCIOLOGÍA DE LAS ORGAMIZACIOMES. McGraw-Hill, Madrid, 1990.

SAMKAR, Y. MAMAGMEMT TECHMOLOGICAL CHAMGE. John Wiley \& Sons, Inc., Mew York, 1991.

SELZMICK, P. LEADERSHIP IM ADMIMISTRATIOM. Harper \& Row, New York, 1957.

SCOTT, R. ORGAMIZATIOMS. RATIOMAL, MATURAL, AMD OPEM SYSTEMS. Prentice-Hall, Inc, New Jersey, 1992.

TAYLOR, F. THE PRIMCIPLES OF SCIEMTIFIC MAMAGEMENT. Harper, New York, 1911.

THOMPSOM, J. D. ORGAMIZACIOMES EM ACCIÓM. McGraw-Hill, Inc., Bogotá, 1993.

WEBER, M. "Bureaucracy". en: M. WEBER, Essays in Sociology. Oxford Univ. Press, New York, 1946.

WIENER, M. CIBERMÉTICA Y SOCIEDAD. Editorial Sudamericana, S. A., Buenos Aires, 1969.

WOODWARD, J. IMDUSTRIAL ORGAMIZATIOM: THEORY AMD PRACTICE. Oxford University Press, London, 1965. 